\title{
A Fuzzy based Routing Protocol for Delay Tolerant Network
}

\author{
Neelam Malik ${ }_{1}$, Shailender Gupta 2 and Bharat Bhushan 3 \\ Electronics and Communication Engineering \\ YMCA University of Science and Technology, Faridabad, INDIA \\ malikneelam.2007@gmail.com 1 ,shailender81@gmail.com 2 ,bhrts@gmail.com 3
}

\begin{abstract}
Delay Tolerant Network (DTN) is a wireless ad hoc network that intends to provide communication between pair of nodes in spite of having intermittent connectivity and long delays. The nodes are buffer and energy constrained. To provide communication in such scenario, a sturdy routing mechanism is needed. In this paper we propose a new routing technique based on fuzzy logic for intermittently connected network. Also an effort has been made to evaluate the performance of the proposed routing strategy in realistic environment (having obstacles) in MATLAB. The simulation results show that the performance of the network is improved using fuzzy logic based routing strategy and the results of realistic environment are quite different from idealistic environment.
\end{abstract}

Keywords: Fuzzy Logic Controller, Realistic Environment, Routing, Rule Bas, Simulation.

\section{Introduction}

The field of DTN has witnessed tremendous development [1-3] in the recent times. The network promises to provide communication between a pair of nodes in spite of frequent interruptions in route and long delays. The nodes in DTN are characterized by mobility, limited battery power and limited buffer storage. Due to this reason it is still a challenging task for designer to design a robust routing protocol which can deliver desired results even in such an environment. Naturally routing in DTN is quite different from routing in ordinary wireless network. The fundamental technique used in any routing technique to deliver the packet from source to the destination is store-carry-forward [4]. However this technique is quite difficult to implement and achieve a stable end-to-end communication path. Many researchers have designed many routing protocols for DTN [5-8] which can be broadly characterized into two categories as follows:

\subsection{Deterministic Routing}

Under this routing protocol [9], future movement and connection are completely known i.e. entire network topology is known in advance or at least is predictable accurately. Each node knows its future neighbouring nodes and their distances so that transmission can be scheduled ahead of time optimally. It is further characterized into three types[10-11] are:

1. Tree Approach

2. Space and Time Approach

3. Modified Shortest Path Approach

\subsection{Stochastic Routing}

In such routing protocols [9], the network behaviour is random and not known. Each node resends the received message with some probability. This protocol depends on decision as to where and when to forward the message to neighbouring nodes. The 
simplest decision is to forward the packet to any neighbour within range, while other decisions are based on history, mobility pattern or any other similar information. It is further characterized into five types [12-17] are:

1. Epidemic or Random Forwarding Approach

2. Prediction or History Based Approach

2.1. Per contact routing based on one hop information only.

2.2. Per contact routing based on average end to end information.

3. Model based routing approach

4. Control Movement

5. Coding Based Approaches

All the above mentioned routing protocols have been proposed to increase the packet delivery ratio under given constraints. The performance of the routing protocol depends upon a number of factors i.e. intermittent connectivity [18], availability of network resources [19-20] like residual energy, buffer availability [21-22], mobility etc.

This paper proposes a new fuzzy logic based routing strategy that tries to maximize packet delivery ratio under the above mentioned constraints. It selects a path for communication between a source and a destination with better delay performance and lesser probability of disconnection. For this purpose a simulator is designed in MATLA 7.0. to evaluate the performance of DTN. Also the performance of the DTN is evaluated in realistic environment [24] i.e. in an environment having obstacles [25] and cyclic nodes. The cyclic nodes are the ones which exhibit cyclic movement on a fixed trajectory and cyclically move in and out of a boundary of simulation region.

The rest of the paper is organized as follows: Section 2 provides literature survey and problem identification. Section 3 gives the proposed routing scheme. Section 4 gives the algorithm and simulation set up parameters. Section 5 illustrates the simulation results. Section 6 present the conclusion followed by the references.

\section{Literature Survey and Problem Identification}

The literature has many papers that discuss the impact of fuzzy based routing scheme on DTN performance in idealistic environment as follows:

Chenn-Jung Huang[26] proposed a fuzzy logic-based edge server selection method for heterogeneous DTN. The experimental results show that the proposed prediction based DTN routing protocol efficiently deliver messages with limited buffer space. The performance metrics used are: the data delivery ratio, average delay and transmitted bytes. The main limitation of the proposed scheme is that the server needs more storage space to record online user history log data and this might increase maintenance costs.

J.D.Mallapur[27]proposed a scheme of Minimum Spanning Tree (MST) by employing a fuzzy controller. The novelty of the proposed scheme is based on MST construction by using acceptable links computed by employing fuzzy controller that considers fuzzy input parameters: link bandwidth, link delay and link reliability. It was observed from the simulation results that the proposed scheme performs better than traditional MST while improving the packet delivery ratio and packet delays. But the main limitation of the scheme is that it does not consider the effect of the mobility of the nodes.

JadMakhlouta [28] improves the probability of delivery and latency by designing a routing protocol based upon fuzzy logic circuit i.e Adaptive Fuzzy Spray and Wait.Work has been done on the buffer prioritization levelusing fuzzy decision making technique which is used to classify messages into levels inside the buffer and promote the messages of high priority level during contact times. The performance of the routing in realistic scenario is not evaluated.

PayamNabhani [29] proposed a new routing scheme in order to dynamically select the relaying node from the available node list. The fuzzy logic system takes input as bandwidth, energy of the node, priority of the message and density of the network based 
upon which the optimized path is selected. The simulation results of the proposed AFRON protocol show that it reduces the energy consumption per transmission with less use of resources. But the above protocol does not take into consideration the realistic scenario.

In all the above mentioned routing strategies for DTN either the effect of realistic environment or exhaustive set of parameters have not been considered. It is therefore needed to design a new routing scheme that cover maximum number of parameters and improves the performance of the network in idealistic as well as in realistic environment. In this paper an effort has been made to combine the different parameters required in the routing decision in order to design a new routing scheme based on Fuzzy Logic Controller (FLC) that improve the efficacy of the DTN. Using FLC, an Optimized path is selected that improves the performance metrics of the network in comparison to its results using Dijikstra shortest path routing protocol in DTN. Besides this the performance of the new routing strategy is also evaluated in the realistic environment. In the next section the proposed scheme is described:

\section{The Proposed Routing Scheme}

The new proposed routing scheme is designed using FLC[30]. Fig.1 shows the block diagram of FLC.

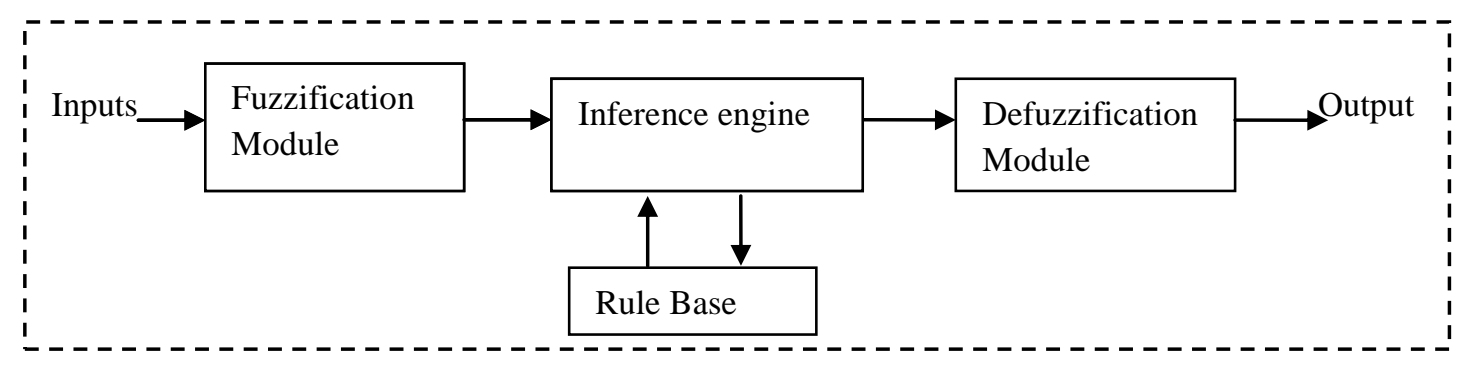

Figure 1. Block Diagram of FLC

FLC are based on expert system, which employs fuzzy logic. Fuzzy logic has two extreme values: either true(1) or false (0).FLC controls with the help of rules and collection of rules is called a rule base. Different blocks of FLC are explained as under:

Fuzzification Module: This module converts each crisp input into a fuzzy set on the domain of the input variable. The fuzzifier performs the fuzzification function that converts the inputs into suitable linguistic values which are needed in the inference engine. Different types of fuzzifier are available, some of which are: singleton fuzzifier, triangle fuzzifier, Gaussiansfuzzifier etc.

Rule Base: It contain a collection of rules in the format of ' IF-THEN' where the 'IF'side of the rule is called antecedent and the 'THEN'side is called the consequent.

Inference Engine:An inference engine is a computer program that tries to derive the answer from rule base. The program used to calculate the result in inference engine is mamdani-type.

Defuzzification Module: This module converts a fuzzy set into crisp set. There are several methods available for defuzzifications: Mean-of-maxima method, centre of gravity method, modified CoG method, Height method etc. 


\subsection{Proposed Model:}

A new fuzzy logic based routing scheme is designed using FLC. Fig.2shows the block diagram of proposed model.The new scheme is designed based upon four input parameters i.e. residual energy, buffer availability, message transmission count (MTC)and fast moving nodes(FMN). These four parameters are applied as input to two FLC's FLC1 \& FLC2. The output of two FLC's becomes the inputs of third FLC and gives a final outputoutput3. Based upon the values of these four inputs for all possible paths FLC3 gives an output3. The maximized value of output 3 provides us an optimized path that will ensure improved performance of the network. The three FLC used are described as follows:

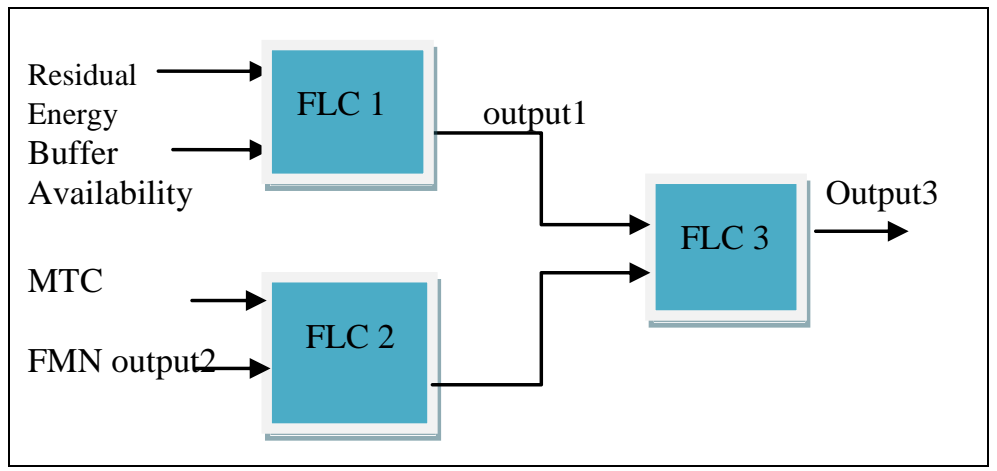

Fig.2 Block Diagram of New Fuzzy Based Routing Strategy

\subsubsection{FLC1}

In this FLC we provide residual energy and buffer availability of nodes as input and obtained an outputoutput 1 of a path using rule base as shown in Fig.3. The membership function for inputs of FLC1 is shown in Fig.4 and Fig.5.

\subsubsection{Residual Energy}

The energy is required during transmission of packet from one node to another node. Initially every node is allocated very high residual energy. If the energy level of the node is $80-100 \%$, it is assumed to have full capacity for communication. The node will not be a good choice to forward the packets if the energy of it falls below 4 percent. For residual energy we define five energy levels:Very Low, Low, Medium, High and Very High fuzzy set as shown in Fig.4.

\subsubsection{Buffer Availability}

According to our proposed scheme each node has buffer in order to store and forward the data during communication. Buffer size availability is divided into five sections Very Low, Low, Medium, High, and Very High. The path which has maximum Buffer availability for the communication is good candidate for forwarding the data between a source and a destination.

\begin{tabular}{|l|l|l|}
\hline $\begin{array}{l}\text { IFBuffer } \\
\text { Availability }\end{array}$ & $\begin{array}{l}\text { IFResidual } \\
\text { Energy }\end{array}$ & THENOutput1 \\
\hline Very Low & Very Low & Very Low \\
\hline Very Low & Low & Very Low \\
\hline Very Low & Medium & Very Low \\
\hline Very Low & High & Low \\
\hline Very Low & Very High & Low \\
\hline Low & Very Low & Very Low \\
\hline
\end{tabular}




\begin{tabular}{|l|l|l|}
\hline Low & Low & Low \\
\hline Low & Medium & Low \\
\hline Low & High & Medium \\
\hline Low & Very High & Medium \\
\hline Medium & Very Low & Very Low \\
\hline Medium & Low & Low \\
\hline Medium & Medium & Medium \\
\hline Medium & High & Medium \\
\hline Medium & Very High & High \\
\hline High & Very Low & Low \\
\hline High & Low & Medium \\
\hline High & Medium & High \\
\hline High & High & High \\
\hline High & Very High & Very High \\
\hline Very High & Very Low & Low \\
\hline Very High & Low & Medium \\
\hline Very High & Medium & High \\
\hline Very High & High & Very High \\
\hline Very High & Very High & Very High \\
\hline
\end{tabular}

Figure 3. Rule Base of FLC 1

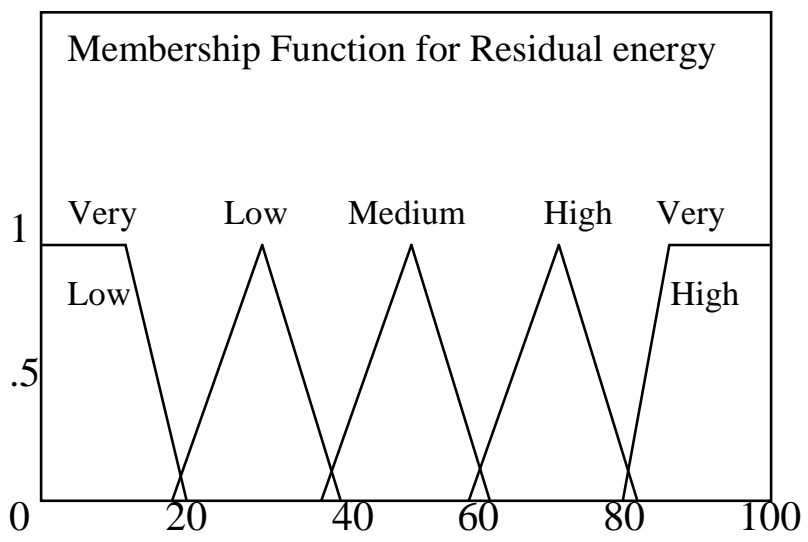

Figure 4. Membership Function for Residual Energy

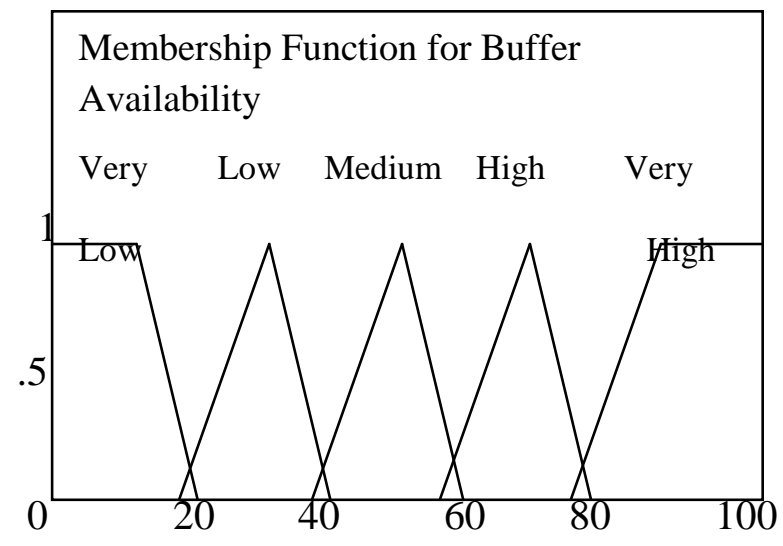

Figure 5. Membership Function for Buffer Availability

\subsubsection{FLC 2}

In this FLC the Message Transmission Count and Fast Moving nodes of a path are given as input. The output output2for that path is derived using rule base shown in Fig.6. The membership functions for inputs of FLC2are shown in Fig.7 andFig.8. The input parameters MTC and FMN are described below:

\subsubsection{Message Transmission Count (MTC)}

Message Transmission Count gives the number of hops from a source through which the message is encountered before reaching to its destination. The membership function of MTC is divided into four fuzzy sets: Low, Medium, High and Very High as shown in Fig.7. 


\subsubsection{Fast Moving Nodes (FMN)}

In the proposed routing scheme, nodes are divided into two categories: slow and fast moving. Both slow and fast moving nodes may move either periodically or nonperiodically in the deployed region. The nodes moving with high speed are divided into four fuzzy sets: Low, Medium, High and Very High as shown in Fig.8.

\begin{tabular}{|l|l|l|}
\hline IF MTC & IF FMN & $\begin{array}{l}\text { THENOutput } \\
2\end{array}$ \\
\hline Low & Low & Very High \\
\hline Low & Medium & Low \\
\hline Medium & Low & High \\
\hline Medium & Medium & Low \\
\hline Medium & High & Low \\
\hline High & Low & High \\
\hline High & Medium & Medium \\
\hline High & High & Low \\
\hline High & Very High & Low \\
\hline Very High & Low & Very High \\
\hline Very High & Medium & Medium \\
\hline Very High & High & Low \\
\hline Very High & Very High & Low \\
\hline
\end{tabular}

Figure 6. Rule Base of FLC 2

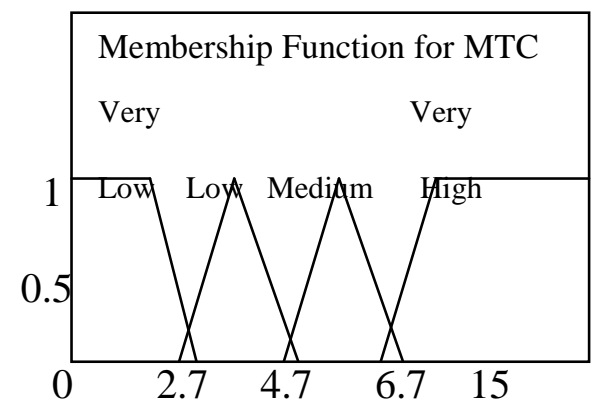

Figure 7. Membership Function for MTC

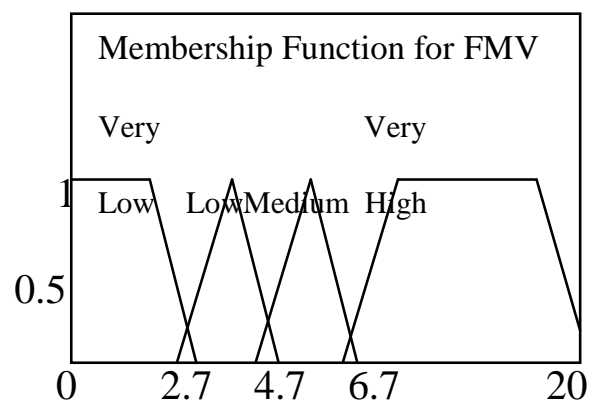

Figure 8. Membership Function for FMV

\subsubsection{FLC 3}

In this FLC the inputs are Output 1 and Output 2 of FLC1 and FLC2 respectively and give output as Output3 for that path based on rule base as shown in Fig.9. The membership function for inputs of FLC3 is shown in Fig.10 and Fig.11.

\begin{tabular}{|l|l|l|}
\hline IF Output1 & IFOutput2 & THEN Output 3 \\
\hline Very Low & Low & Low \\
\hline Very Low & Medium & Low \\
\hline Very Low & High & Low \\
\hline Very Low & Very High & Medium \\
\hline
\end{tabular}




\begin{tabular}{|l|l|l|}
\hline Low & Low & Low \\
\hline Low & Medium & Low \\
\hline Low & High & Low \\
\hline Low & Very High & High \\
\hline Medium & Low & Low \\
\hline Medium & Medium & Medium \\
\hline Medium & High & Medium \\
\hline Medium & Very High & High \\
\hline High & Low & Low \\
\hline High & Medium & Medium \\
\hline High & High & Medium \\
\hline High & Very High & Very High \\
\hline Very High & Low & Medium \\
\hline Very High & Medium & Medium \\
\hline Very High & High & High \\
\hline Very High & Very High & Very High \\
\hline
\end{tabular}

Figure 9. Rule Base of FLC 3

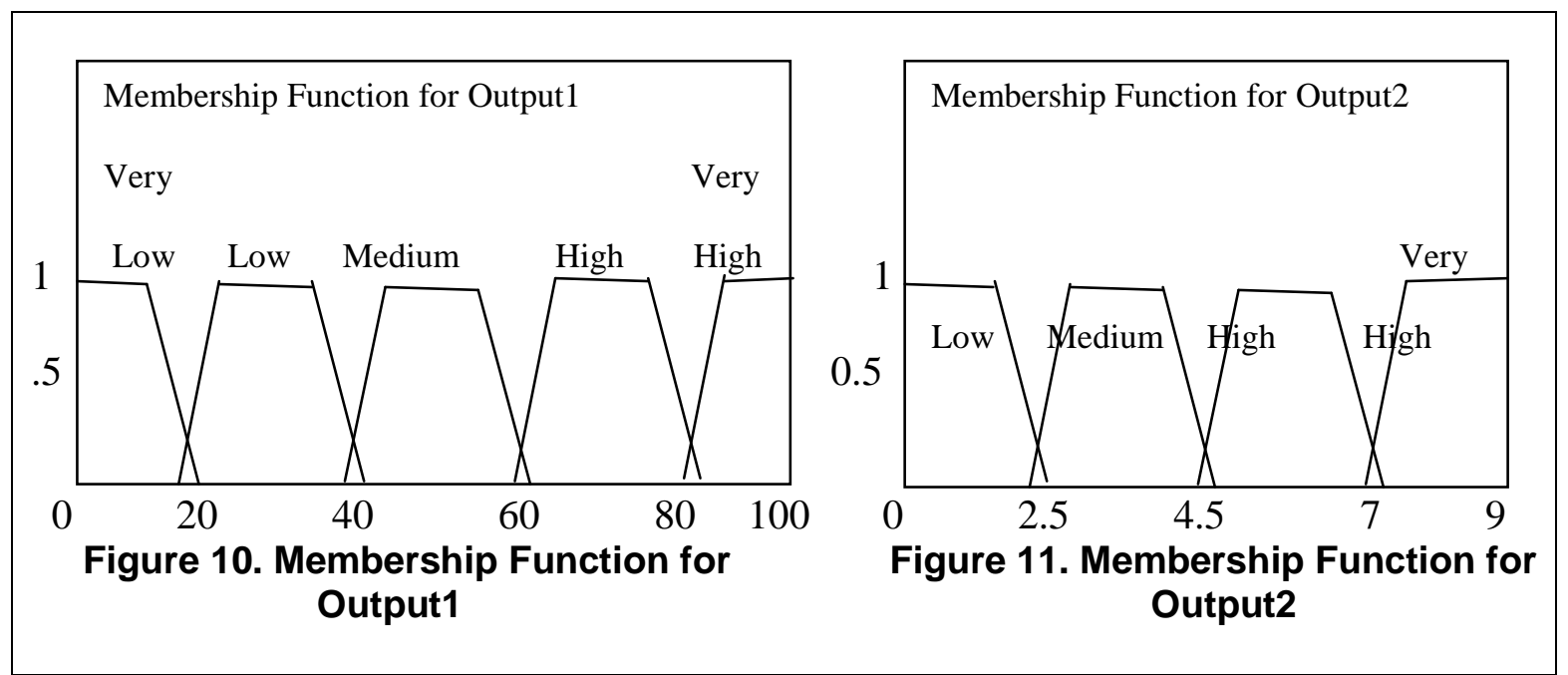

\section{Experimental Set Up}

For the implementation of the proposed fuzzy logic based routing strategy for DTN a simulator was designed in MATLAB-7.0. The simulation region is of size 1500x1500 of square shape is termed as inner region and region of width 500 units is drawn around inner region termed as outer region. In the inner region forty number of nodes were distributed randomly. Dijkstra's shortest path algorithm and new fuzzy based routing strategy were used to provide communication path between a source and a destination at a given time. Fig. 12 and Fig.13 show the snapshot of simulation process in idealistic and realistic environment respectively, where green lines represent the communication path using new fuzzy based routing scheme whereas lines in red colour represents path formed by using dijkstra shortest path routing. In Fig.13 green colour shaded region show the presence of obstacles in realistic scenario. Out of 40 nodes taken some nodes are designated as cyclic nodes and are represented by red dots that may moves in and out of inner periphery cyclically but never go out of outer periphery. 


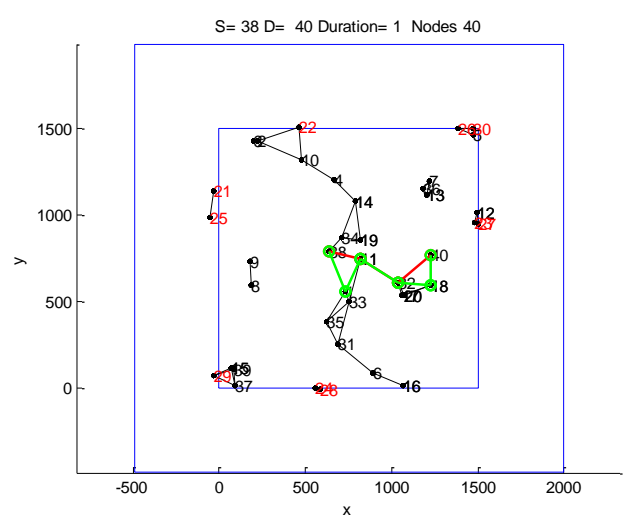

Figure 12. Snapshot of the DTN in Idealistic Environment

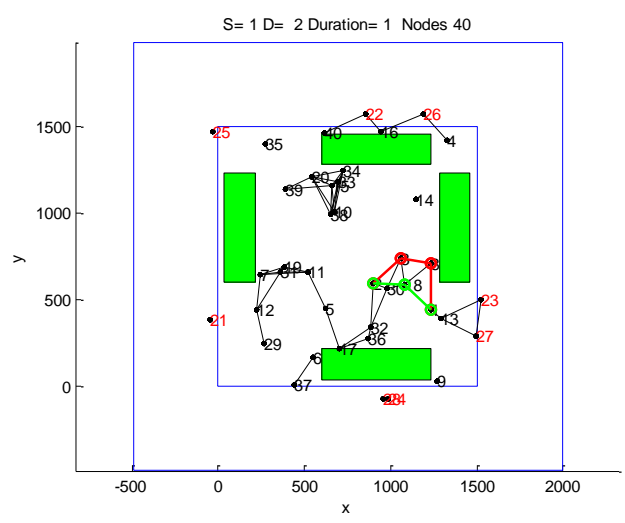

Figure 13. Snapshot of the DTN in Realistic Environment

\subsection{Set up Parameters}

The table 1 shows the values of set up parameters used for simulation purpose.

Table 1. Set Up Parameters

\begin{tabular}{|c|c|c|c|}
\hline Set up parameter & Value & Set up parameter & Value \\
\hline $\begin{array}{l}\text { Inner region } \\
\text { dimension }\end{array}$ & $\begin{array}{l}1500 \times 1500 \\
\text { sq units }\end{array}$ & $\begin{array}{l}\text { Speed of slow DTN } \\
\text { nodes }\end{array}$ & $1 \mathrm{~m} / \mathrm{s}$ \\
\hline $\begin{array}{l}\text { Outer region } \\
\text { dimension }\end{array}$ & $\begin{array}{l}2500 \times 2500 \\
\text { sq units }\end{array}$ & Routing algorithm's & $\begin{array}{l}\text { Dijkstra's Shortest Path and } \\
\text { Fuzzy based routing strategy }\end{array}$ \\
\hline Numbers of nodes & 40 & $\begin{array}{l}\text { Packet transmission } \\
\text { interval }\end{array}$ & $1 \mathrm{sec}$ \\
\hline Transmission range & $275 \mathrm{~m}$ & Packet Size & 512 bytes \\
\hline Mobility Model & $\begin{array}{l}\text { Random } \\
\text { Walk }\end{array}$ & $\begin{array}{l}\text { Number of packet } \\
\text { sent }\end{array}$ & 20 \\
\hline $\begin{array}{l}\text { Speed of fast DTN } \\
\text { nodes }\end{array}$ & $7 \mathrm{~m} / \mathrm{s}$ & Number of FLC & 3 \\
\hline Placement of nodes & Random & $\begin{array}{l}\text { Area of each } \\
\text { obstacles }\end{array}$ & 112500 sq units \\
\hline Obstacles shape & Rectangle & Shape of periphery & Square \\
\hline
\end{tabular}




\subsection{Algorithm}

The algorithm to calculate the various performance metrics for DTN is shown in Algorith 1 . In the algorithm total forty nodes $(N=40)$ were deployed and $k \%$ of nodes defined as DTN nodes [31]. To calculate the value of PoR a variable called count is used to find the total no of paths that exists between all S-D pairs. If the path exists between S$\mathrm{D}$ pair, the value of count variable is incremented by 1 . For calculating the value of average hop count using shortest path routing and new fuzzy based routing scheme the Cum_Hop_count_shortest and Cum_Hop_count_fuzzyvariable is used respectively. If the path exists between pair of S-D then again check if path is intersected by an obstacle or not. If the path is not intersecting by the obstacle then the value of hop count is added to Cum_Hop_count_shortest and Cum_Hop_count_fuzzy variable using both routing scheme. This process is repeated for all combinations of S-D pairs. For calculating PDR the source sends 20 packets using procedure send_data() between every S-D pair and returns successfully packets received by destination. A variable called Cum_Data_packet_shortest and Cum_Data_packet_fuzzy is used to find cumulative value of packet received by destination using shortest path routing and new fuzzy based routing scheme.Path_length_shortest and Path_length_fuzzycontains the distance between source and destination thru intermediate nodes using both routing schemes. If a path is broken due to intermediate cyclic node going out of range then there is possibility of again formation of the same path.Delay_fuzzy and Delay_shortest calculate the delay between break and remake of the same path. The average hop count, PoR and packet delivery ratio are calculated by using formula given in algorithm.

Algorithm 4.2: To Calculate Various Performance Metrics

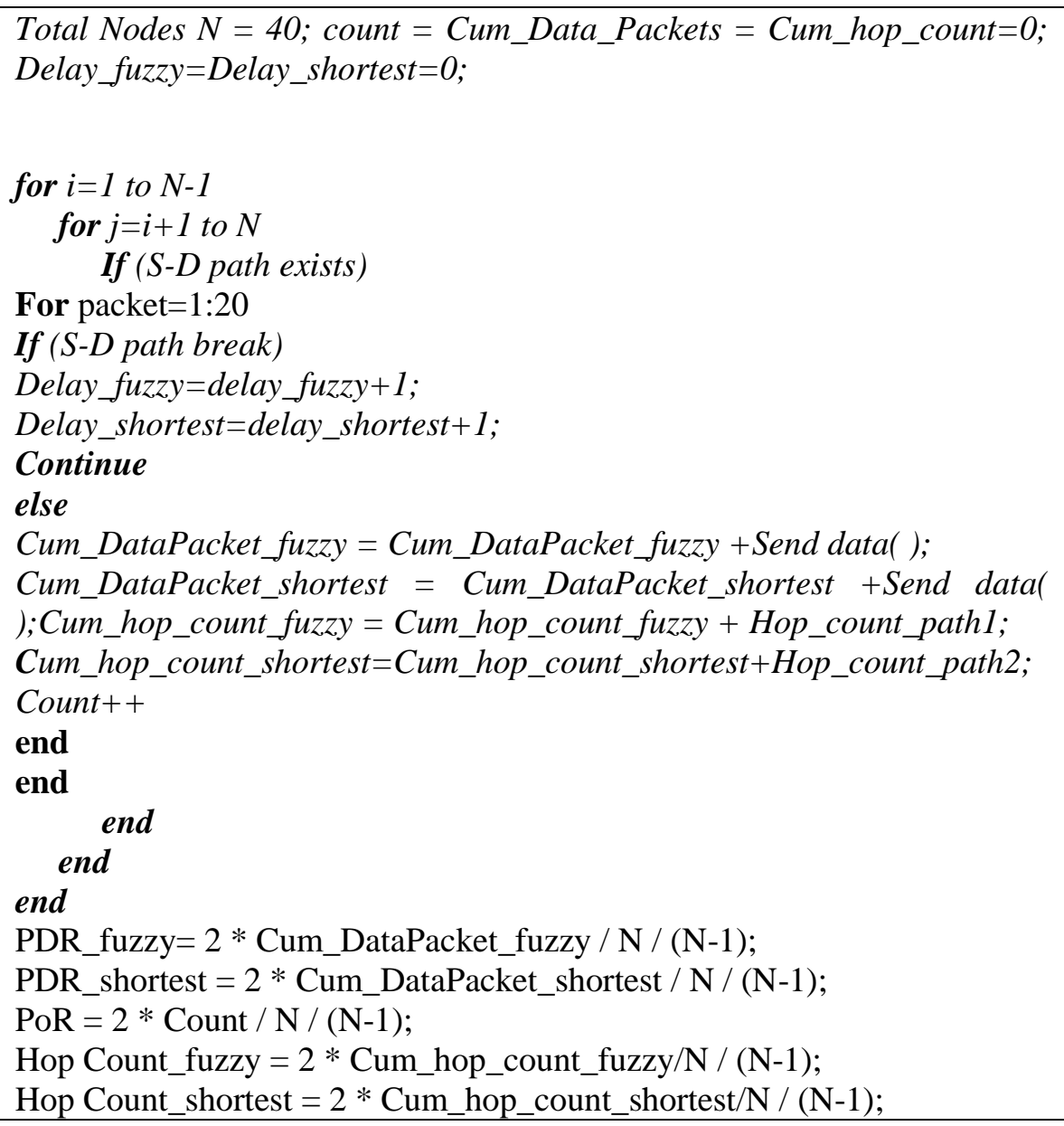




\title{
4.3 Performance Metrics
}

The following parameters are used as performance metrics for DTN:

4.3.1 Packet delivery ratio (PDR)[32]:defined as the number of packets received by the destination to the total number of packets sent by the source.

4.3.2 Hop count[33]:defined as the number of intermediate nodes required to establish the path from source to destination.

4.3.3 Probability of reachability (PoR)[34]:defined as fraction of possible reachable routes to all possible routes that may physically exist between every pair of source and destination.

4.3.4 Delay[35]:defined as the time duration in which a broken path is reformed.It is an interval between a path broken and again reformation of the same path.

\section{Simulation Results}

\subsection{Impact on Packet Delivery Ratio (PDR)}

Fig.14 shows the impact on PDR using proposed routing and shortest path routing in both idealistic and realistic environment. The following inference can be drawn from the graph:

- The proposed strategy increases the value of PDR in comparison to its value using shortest path routing protocol using both idealistic and realistic scenario.It is due to reason that the optimized path using fuzzy method has lesser probability of disconnection in comparison to the shortest path.

- As the percentage of cyclic nodes increases the value of PDR decreases continuously.

- The presence of obstacles results in decrease in value of PDR using either strategy.

\section{PDR}

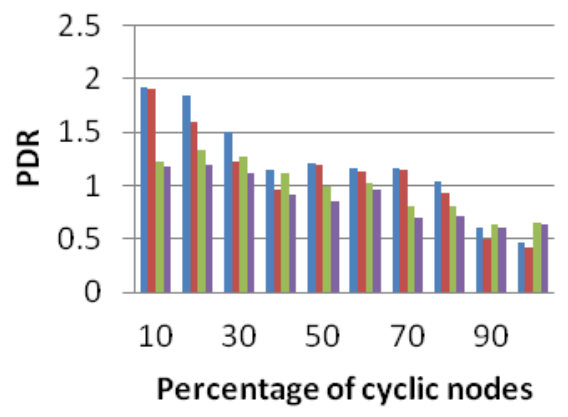

\author{
Proposed \\ scheme Idealistic \\ Shortest \\ path_Idealistic \\ Proposed \\ scheme_Realistic \\ Shortest \\ path_Realistic
}

\section{Figure 14. Impact on PDR}

\subsection{Impact on Hop Count}

Fig.15 shows the comparison of impact of using proposed routing scheme and shortest path routing scheme on hop count in both idealistic and realistic environment. The following inference can be made from the graph:

- The value of hop count in the fuzzy based strategy is larger than the value of hop count for shortest path routing strategy in the same scenario (idealistic or 
realistic).The reason for the same is that the path length of the optimized path is larger in comparison to the shortest path. The other reason may be that the optimized path contains large number of slow moving nodes than the shortest path that reduces the chance of disconnection of optimized path.

- As the percentage of cyclic nodes increases the value of hop count decreases.

- The presence of obstacles in realistic environment results in increase of value of hop count because obstacle results in increases in path length

\section{Hop Count}

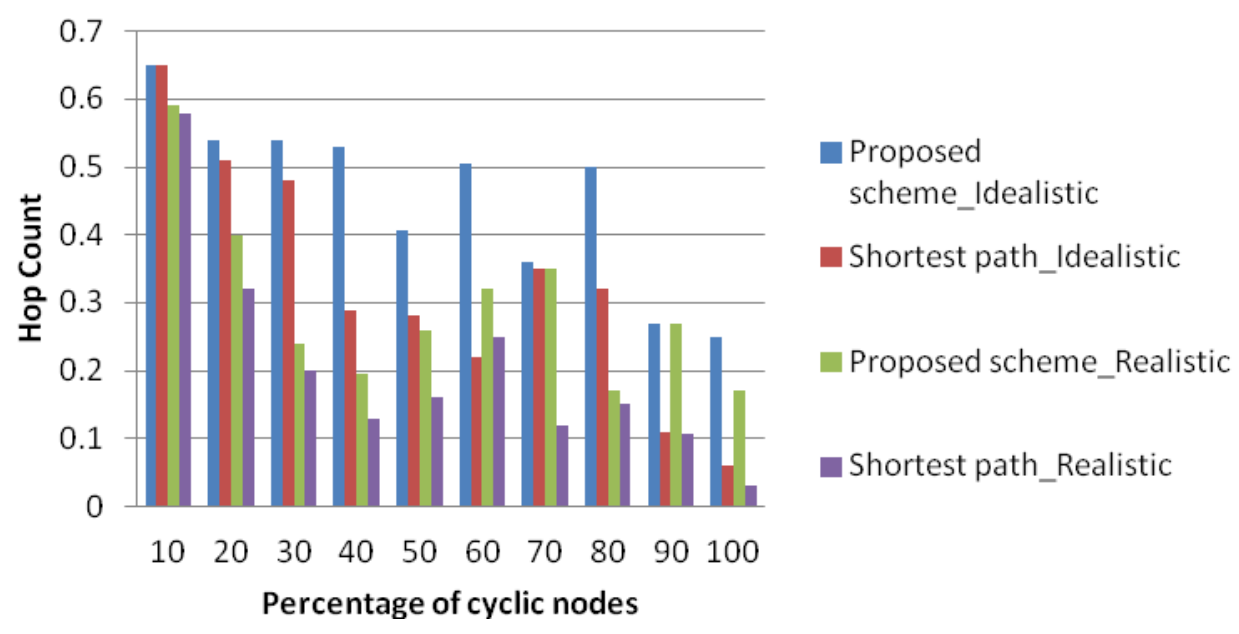

Figure 15. Impact on Hop Count

\subsection{Impact on Probability of Reachability (PoR)}

Fig.16 shows the impact on PoR using proposed routing scheme and shortest path routing scheme in both idealistic and realistic environments. The following inference can be made from the graph:

- The value of PoR in idealistic scenario is higher than the value of PoR in realistic scenario. This is due to the hindrance provided by the obstacles to the signal between neighbouring nodes. Hence lesser number of reachable routes is formed in realistic environment compared to its value in the idealistic environment.

- The value of PoR decreases as the percentage of cyclic nodes increases. With increase in percentage of cyclic nodes, more number of nodes may move out of inner periphery that may lead to disconnection of the path. 


\section{PoR}

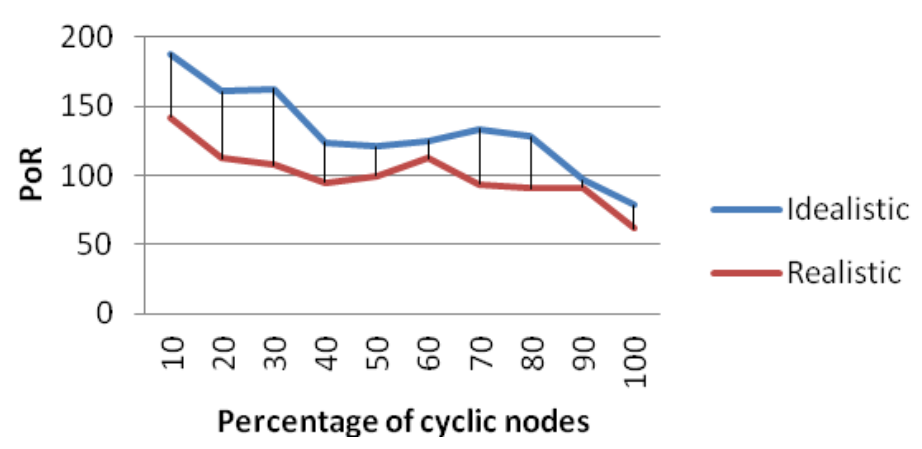

Figure 16. Impact on PoR

\subsection{Impact on Delay}

Fig.17shows the impact on delay introduced during communication using proposed scheme and shortest path scheme in both idealistic and realistic environments. The following inference can be made from the graph:

- As the percentage of cyclic node increases delay in the communication increases. This is due to the fact that the possibility of reformationof the broken path due to the presence of cyclic nodes increases.

- Using proposed fuzzy logic strategy, the value of delay introduced in the communication path is lesser than the delay introduced using shortest path routing strategy. This shows that using shortest path routing there is lesser number of completion of data transfer takes place in comparison to proposed fuzzy logic based strategy.

- The value of delay in realistic environment is more than its value in idealistic environment. This is due to presence of obstacles. More number of paths are likely to break and there is more likelihood of reformation of the broken path that increases the delay in data transmission.

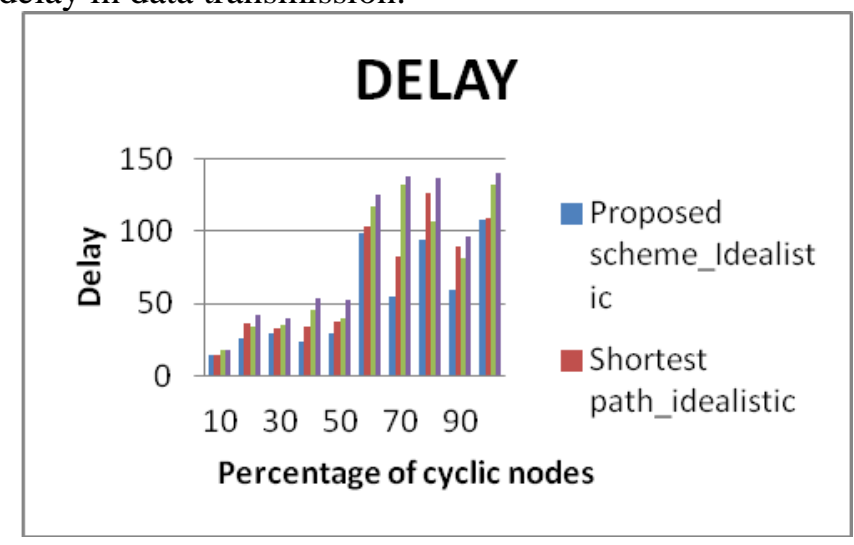

Fig.17 Impact on Delay

\section{Conclusion}

In this paper an effort has been made to design a new routing scheme based on fuzzy logic for DTN and compare its performance with shortest path routing technique in an idealistic environment and realistic environment. The following inference may be drawn from the results: 
- The performance metrics of DTN using proposed fuzzy logic based strategy improves the performance of the network over its performance using shortest path routing strategy.

- The performance of the network degrades gradually due to the presence of the obstacles using both routing strategies. This shows that the idealistic results cannot be used for practical applications.

- The network performance decreases as the percentage of cyclic nodes increaseusing both strategies in idealistic and realistic scenarios.

\section{References}

[1] Q. H. Li, S. C. Zhu and G. H. Cao, "Routing in Socially Selfish Delay Tolerant Networks", Department of Computer Science \& Engineering, The Pennsylvania State University, University Park, INFOCOM, Proceedings IEEE, (2010).

[2] A. Krifa, C. Barakat and T. Spyropoloulos, "Optimal Buffer Management Policies for Delay Tolerant Networks", IEEE SECON, (2010).

[3] V. Conan, J. Leguay and T. Friedman, "Fixed Point Opportunistic Routing in Delay Tolerant Network", IEEE journal on Selected Areas in Communications, vol. 26, no. 5, (2008), pp. 773 - 782.

[4] N. Wisitpongphan, F. Bai, P. Mudalige, V. Sadekar and O. Tonguz, "Routing in sparse vehicular ad hoc wireless networks”, IEEE J. Sel. Areas Commun., vol. 25, no. 8, (2007), pp. 1538-1556.

[5] P. U. Tournoux, J. Leguay, F. Benbadis, V. Conan, M. Amorim and J. Whitbeck, "The Accordion Phenomenon: Analysis, Characterization, and Impact on DTN Routing", Proceeding of infocom, IEEE, (2009).

[6] B. Divecha, A. Abraham, C. Grosan and S. Sanyal, "Impact of Node Mobility on MANET Routing Protocols Models", Journal of Digital Information Management, vol. 5, no. 1, (2007), pp. 19-24.

[7] L. Yin, H. M. Lu, Y. D. Cao and J. M. Gao, "Cooperation in Delay Tolerant Network", School of Computer Science, Beijing Institute of Technology, Beijing, PRC, INFOCOM, Proceedings IEEE, (2010).

[8] X. Chen, J. Shen and J. Wu, "Improving routing protocol performance in delay tolerant networks using extended information", The Journal of Systems and Software, vol. 83, (2010), pp. 1301-1309.

[9] Z. S. Zhang, "Routing In Intermittently Connected Mobile Ad Hoc Networks and Delay Tolerant Networks: Overview and Challenges", San Diego Research Center, 1st Quarter IEEE Communications the Electronic Magazine of Original Peer-Reviewed Survey Articles, pp. 24-37.

[10] S. Merugu, "Routing in Space and Time in Network with Predicable Mobility", Georgia Institute of technology, technical report, GIT-CC-04-7, (2004).

[11] S. Jain ,K. Fall and R Patra, "Routing in Delay Tolerant Network", Proceeding of ACM SIGCOMM, (2004).

[12] E. P.C. Jones, L. Li, K. Jakub, P. Schmidtke and A. S. Ward. "Practical Routing in Delay-Tolerant Networks", IEEE Transactions on Mobile Computing, vol. 6, no. 8, (2007).

[13] Y. Wang, S. Jain, M. Martonosi and K. Fall, "Erasure Coding Based Routing forOpportunistic Networks", in Proceedings of ACM SIGCOMM Workshop on Delay Tolerant Networking (WDTN), (2005).

[14] Q. Li and D. Rus, “Communication in Disconnected Ad hoc Network using message relays”, J. Parallel Distributed computing, vol. 63, (2003), pp. 75-86.

[15] Z. Chen, "Ad hoc realy wireless network over moving vehicles on highways", ACM Mobihoc, (2001).

[16] P. Juang, "Energy-Efficient Computing for Wildlife Tracking: Design Tradeoffs and Early Experiences with Zebranet", Proc. ASPLOS, (2002).

[17] A. Vahdat and D. Becker, "Epidemic Routing for Partially Connected Ad hoc Networks", Duke University, Tech. Rep.CS-200006, (2000).

[18] G. Resta and P. Santi, "A Framework for Routing Performance Analysis in Delay Tolerant Networks with Application to Non Cooperative Networks", proceedings of the 6th IEEE conference on Sensor, mesh and ad hoc Communications and Networks (SECON), (2009).

[19] J. D. Mallapur, S. S. Manvi and D. H. Rao, "A Fuzzy Based Approach for Multicast Tree Computation in Wireless Multimedia Networks", Journal of information and computing science, vol. 4, no. 2, (2008), pp. 83-92.

[20] V. Mahendran, S. K. Anirudh, C. Siva and R. Murthy, "A Realistic Framework for Delay-Tolerant Network Routing in Open Terrains with Continuous Churn", Proceedings of the 12th international conference on Distributed computing and networking, ICDCN'11.

[21] M. Radenkovic and A. Grundy, "Efficient and adaptive congestion control for heterogeneous delaytolerant networks", Elsevier journal, vol. 10, no. 7, (2012), pp. 1322-1345.

[22] P. Nabhani and A. M. Bidgoli, "Adaptive Fuzzy Routing in Opportunistic Network", International Journal of Computer Applications, vol. 52, no. 18, (2012). 
[23] S. C. Lo and C. L. Lu, "A Dynamic Congestion Control based Routing for Delay-Tolerant Networks", Fuzzy Systems and Knowledge Discovery (FSKD), 9th International Conference.

[24] S. Gupta, C. Kumar, C. K. Nagpal and B. Bhushan, "Performance Evalaution of MANET in Realistic Environment", I. J. Modern Education and Computer Science, vol. 7, (2012), pp. 57-64.

[25] C. Kumar, C. K. Nagpal, B. Bhushan and S. Gupta, "Towards Realistic Performance Evaluation of Mobile Ad Hoc Network", World Congress on Information and Communication Technologies (WICT), pp. 287 - 291.

[26] C. J. Huang, H. M. Chen, C. F. Lin, J. J. Liao, K. W. Hu, D. X. Yang, C. H. Chen and Y. T. Chuang, “A Scalable Routing for Delay-Tolerant Heterogeneous Networks", International Journal of Innovative Computing, Information and Control ICIC International, vol. 8, no. 1, (2012), pp. 49-59.

[27] J. D. Mallapur, S. S. Manvi and D. H. Rao, “A Fuzzy Based Approach for Multicast Tree Computation in Wireless Multimedia Networks", Journal of Information and Computing Science, vol. 4, no. 2, (2009), pp. 083-092.

[28] J. Makhlouta, H. Harkous, F. Hutayt and H. Artail, "Adaptive Fuzzy Spray and Wait: Efficient Routing for Opportunistic Networks", IEEE, (2011).

[29] P. Nabhani, A. MasoudBidgoli, "Adaptive Fuzzy Routing in Opportunistic Network", International Journal of Computer Applications, vol. 52, no. 18, (2012).

[30] M. Ganesh, "Introduction to Fuzzy Sets and Fuzzy Logic", PHILearing Private Limited, new delhi, (2009), pp. 169,184-186.

[31] H. G., J. Li and Y. Qian, "HoP-DTN: Modeling and Evaluation of Homing-Pigeon-Based DelayTolerant Networks", IEEE transactions on vehicular technology, vol.59, (2010).

[32] C. Liu and J. Wu, "An Optimal Probabilistic Forwarding Protocol in Delay Tolerant Networks", mobihoc, (2009).

[33] S. Medjiah and T.Ahmed, "Orion Routing Protocol for Delay-Tolerant Networks", IEEE ICC, (2011).

[34] S. Gupta, C. K. Nagpal and C. Singla, "Impact of Selfish node Concentration in Manets", International Journal of Wireless \& Mobile Networks (Ijwmn), vol. 3, no. 2, (2011).

[35] W. Gao, Q. H. Li, B. Zhao and G. H. Cao, "Multicasting in Delay Tolerant Networks: A Social Network Perspective", Mobihoc, pp. 299-308.

\section{Authors}

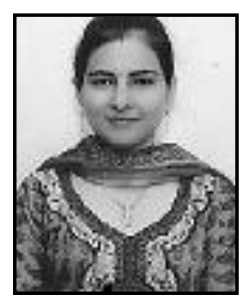

Neelam Malik

Assistant Professor

NIT kurukshetra,kurukshetra 